\title{
OC21 - Psychosocial functioning of parents of infants diagnosed with complex congenital heart defect
}

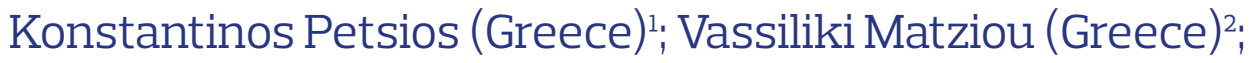 \\ Ioanna Voutoufianaki (Greece) ${ }^{1}$; Anna Manatou (Greece)ㄹ; \\ Prodromos Azariadis (Greece) ${ }^{1}$; Spiros Rammos (Greece) ${ }^{1}$ \\ ${ }^{1}$ 'Onassis' Cardiac Center; ${ }^{2}$ - Faculty of Nursing, National and Kapodistrian \\ University of Athens
}

Theme: Parenting/parenthood.

Keywords: Congenital heart defect (CHD), parenting, psychosocial.

Introduction: The diagnosis of complex CHD impacts the psychosocial status of parents and their functioning.

Purpose: A critical evaluation of the literature concerning the psychosocial parental response to their infant's diagnosis of complex CHD.

Methods: Systematic review of 18 articles published after 2000 in PubMed and CINAHL.

Results: The impact of an infant's CHD on the family functioning is determined both by child's medical condition and family's psychosocial factors. The majority of parents experience intense loss and numerous stressors. Nurses and physicians need to be sensitive to the needs, thoughts and experiences of the parents when discussing treatment options. Families with poor social support networks may have the greatest need for professional interventions.

Conclusions: Appropriate interventions assist the majority of parents to adapt to the diagnosis of complex CHD. However, there are a number of parents with an increased psychosocial risk associated with higher rates of emotional distress.

\section{OC22 - Light House Project}

Gonçalo Pereira (Portugal)i; Ana Baridó (Portugal)²

${ }^{1}$ Universidade Católica Portuguesa - Instituto de Ciências da Saúde - Lisboa;
${ }^{2}$ ACES Leiria

Theme: Accreditation and quality improvement.

Keywords: Adolescents, indicators, nursing care, nursing consultation, quality improvement.

In the context of primary health care internship of a master's degree in paediatric nursing ministered by 'Universidade Católica Portuguesa' in Lisbon, and after diagnosing a 